\title{
Sustentabilidad como una alternativa para alcanzar la seguridad alimentaria
}

\author{
Cecia Martínez ${ }^{1}$ \\ martinezcecia@hotmail.com \\ Ricardo Martínez ${ }^{2}$ \\ richarmtz@hotmail.com
}

\section{RESUMEN}

En México la seguridad alimentaria se convierte en un problema de desarrollo sustentable cuando se carece de los medios y recursos para tener acceso a los alimentos debido a los niveles de hambruna y desnutrición, situación que impide a las personas tengan una vida digna y de calidad; como consecuencia se genera un estado de pobreza. Motivo por el que se abordará, qué es el derecho a la alimentación, en qué consiste la sustentabilidad, y la importancia de la seguridad alimentaria como garantía al derecho a la alimentación.

Palabras claves: alimentación; desarrollo; pobreza; sustentabilidad y seguridad alimentaria.

\footnotetext{
${ }^{1}$ Maestro en Métodos de Solución de Conflictos y Derechos Humanos, PNPC-CONACYT, Universidad Juárez Autónoma de Tabasco, Villahermosa, Tabasco, México, correo electrónico: richarmtz@hotmail.com. Teléfono celular: 993-242-18-47

${ }^{2}$ Maestra en Métodos de Solución de Conflictos y Derechos Humanos, PNPC-CONACYT, Universidad Juárez Autónoma de Tabasco, Villahermosa, Tabasco, México correo electrónico: richarmtz@hotmail.com.Teléfono celular:
} 


\title{
Sustainability as an alternative to achieve food safety
}

\begin{abstract}
In Mexico, food security becomes a problem of sustainable development when there is a lack of means and resources to have access to food due to levels of hunger and malnutrition, a situation that prevents people from having a dignified life and quality; as a consequence a state of poverty is generated. Reason why it will be addressed, what is the right to food, what is sustainability, and the importance of food security as a guarantee of the right to food.
\end{abstract}

Keywords: food; development; poverty; sustainability; and food securyty.

Artículo recibido: 10 Agosto. 2021 Aceptado para publicación: 07. Setiembre. 2021 Correspondencia: martinezcecia@ hotmail.com Conflictos de Interés: Ninguna que declarar 


\section{INTRODUCCIÓN}

El objetivo del presente trabajo consiste en analizar la relevancia de la seguridad alimentaria para poder alcanzar un desarrollo sustentable como protección al derecho humano a la alimentación que tienen los seres humanos tal y como se encuentra regulado en los artículos 4 de la Constitución Política de los Estados Unidos Mexicanos, 25 de la Declaración Universal de Derechos Humanos y el numeral 11 del Pacto de Derechos Económicos, Sociales y Culturales; con el fin de generar conciencia sobre la relación que existe entre los alimentos que se consumen, la salud y las condiciones de vida de los seres humanos.

El Estado debe respetar, proteger, promover y garantizar una alimentación adecuada, sana y de calidad a las personas; algunas de sus obligaciones son de carácter inmediata y otras deben hacerse efectiva de forma gradual, destinando la mayor parte de los recursos disponibles para su cumplimiento. Dentro de las obligaciones de los Estados, referentes al derecho a la alimentación, se regulan en cada instrumento internacional desde perspectivas diferentes, la adopción de medidas para el ejercicio progresivo y pleno de este derecho para todos los individuos.

De igual manera, el Estado debe promover el desarrollo rural integral, con el propósito de generar empleo y brindar a la población campesina el bienestar y participación en el desarrollo nacional. Dentro de sus fines tendrá que garantizar el abasto suficiente y oportuno de los alimentos básicos que la ley establezca (Constitución Política de los Estados Unidos Mexicanos, 2018, artículo 27). Todas las personas deben tener acceso y disponibilidad de los mismos, incluyendo a los niños, niñas y adolescentes, toda vez que, en México, el sobrepeso y la obesidad son problemas de salud pública, donde la transición de enfermedades ha ganado espacio en los últimos años.

Desde el enfoque legislativo, se ubica la Ley Marco Derecho a las Alimentación, Seguridad y Soberanía Alimentaria, misma que ofrece una definición precisa del alcance y contenido del derecho humano a la alimentación. Establece las obligaciones de las autoridades del Estado y el sector privado, los mecanismos institucionales necesarios y proporciona las bases jurídicas y demás medidas esenciales que deberán adoptar las autoridades competentes.

Igualmente, se hace referencia a la iniciativa con proyecto de decreto por el que se expide la Ley General del derecho a la alimentación adecuada, su objetivo es garantizar el 
ejercicio del Derecho a la Alimentación, establecido en el artículo 4, párrafo tercero y sexto y en el párrafo segundo de la fracción vigésima del artículo 27 de la Constitución Política de los Estados Unidos Mexicanos.

Con relación a la legislación que regula la calidad de los alimentos en México, se tiene a la Ley General de Salud, misma que reglamenta el derecho a la protección a la salud que tiene toda persona conforme al artículo 4 de la Constitución Política de los Estados Unidos Mexicanos. Esta ley establece en el artículo 3 que es materia de salubridad general, dentro de ellas: La protección social en salud, el control sanitario de productos y servicios y de su importación y exportación (Ley General de Salud, 2018).

El derecho a la alimentación es un derecho humano, reconocido en la Constitución, instrumentos internacionales y legislaciones, que tienen como fin proteger el derecho de todos los seres humanos a alimentarse con dignidad, mismo que es vulnerado ante la presencia del hambre en la comunidad internacional. La Organización de las Naciones Unidas para la Alimentación y la Agricultura (FAO), como agencia de las Naciones Unidas lidera el esfuerzo internacional para poner fin al hambre (Organización de las Naciones Unidas para la Alimentación y la Agricultura, 2019).

Dicha organización desempeña un papel importante a la hora de promover entornos favorables, implementa legislaciones, mecanismos de gobernanza y orientación técnica en el proceso de formulación de políticas públicas para garantizar que todos los niños, mujeres y hombres, puedan disfrutar de una alimentación, sana, nutritiva y suficiente, tomando medidas a favor de la realización del derecho humano a la alimentación.

\section{ASPECTOS PRINCIPALES DEL DERECHO A LA ALIMENTACIÓN}

La alimentación se entiende como el derecho a alimentarse dignamente. Se considera a la alimentación como una "actividad colectiva tanto por la forma de obtención de sus ingredientes, como por las características de su consumo" (Barriguete, Meléndez, 2017, p. 25). Sin embargo, los cambios en los tipos de alimentación y el abuso del consumo de los alimentos son factores que ponen en peligro a la especie, a ser víctima del hambre y exponer la actividad física relacionada con el trabajo en el campo.

En la lucha contra el hambre juegan un papel importante aquellos movimientos que se han propuesto no sólo alcanzar ayudas a casos urgentes, sino analizar sus causas y denunciar los intereses de fondo que impiden soluciones globales. En el caso mexicano se desarrollaron luchas campesinas en el norte del país, como la de los pueblos yaquis de 
Sonora y la de los raramuris o tarahumaras de chihuahua. En los años de 1905 y 1907 las huelgas mineras y de textiles en Cananea, Sonora y Río Blanco, Veracruz, expresaron las contradicciones socioeconómicas subyacentes a millones de proletariados, campesinos y desheredados de la tierra que anunciaron las revoluciones sociales de carácter proletariado y agrario.

La Revolución Mexicana de 1910 fue un movimiento revolucionario de un pueblo que explotó en contra del gobierno opresor de Porfirio Díaz, gracias a las diferencias en las clases sociales, donde se luchaba por un pueblo con tierra y libertad. Es una de las etapas más trascendentales de la historia de México, que trajo como resultado apreciables cambios sociales.

Ante estos precedentes, la alimentación se entiende como un derecho social que exige la disponibilidad de los mismos en cantidad y calidad para satisfacer las necesidades alimentarias de las personas. El derecho en mención, requiere que las personas tengan la capacidad para obtener los alimentos en cantidades suficientes mediante diversas formas: producción, posesión de tierras, suministro de semillas y agua. Para ello, el Estado tiene la obligación de garantizar el acceso a la alimentación mediante la adopción de medidas que le permitan lograr su pleno ejercicio.

Asimismo, se entiende la alimentación como el derecho a alimentarse dignamente. Considerada como el derecho humano al que tiene acceso cualquier persona por el solo hecho de serlo, está contemplado en diversas referencias nacionales e internacionales. Es un derecho que consiste en tener todos los elementos nutritivos que un individuo necesita para vivir una vida sana y activa, así como los medios para tener acceso a ellos (Naciones Unidas, s.f.). Las personas deben tener una alimentación adecuada, es decir, deben satisfacer las necesidades de dieta de acuerdo a sus características: edad, condiciones de vida, salud, ocupación, etcétera.

Es de menester precisar que no es lo mismo el derecho a la alimentación que el derecho a ser alimentado. El primero consiste en el derecho a alimentarse en condiciones de dignidad. Una persona debe vivir en condiciones que le permitan producir alimentos o comprarlos, mientras que el segundo, señala que es deber del Estado suministrarle alimentación directamente (Naciones Unidas, s.f) a las personas que no pueden hacerlo con sus propios medios. 
Desde el enfoque de la Declaración Universal de los Derechos Humanos, como instrumento internacional, se reconoce la dignidad y la igualdad inherentes a todas las personas y se expresa el derecho que tiene todo ser humano a contar con un nivel de vida adecuado que le asegure, a sí mismo como a su familia, salud y bienestar, en especial la alimentación(Declaración Universal de Derechos Humanos, 1948, artículo 25). Dicha declaración, regula el derecho a gozar de un nivel de vida adecuado y del más alto nivel que necesita un individuo para satisfacer sus necesidades alimentarias.

De esta forma, el derecho a la alimentación comenzó a ser ratificado por otros tratados en materia de derechos humanos, entre los que figuran: el Pacto Internacional de Derechos Económicos, Sociales y Culturales (1966), el Pacto Internacional de Derechos Civiles y Políticos (1966) y sus Protocolos, la Convención Internacional sobre la Eliminación de todas las formas de Discriminación Racial (1965), Convención de (1979) sobre la Eliminación de todas las Formas de Discriminación contra la Mujer, Convención de (1989) sobre los Derechos del Niño, Convención sobre el Estatuto de los Refugiados (1951) y Convención sobre el Derecho de las Personas con Discapacidad (2006).

Por lo que hace al Comité de Derechos Económicos, Sociales y Culturales (Comité de DESC) en su Comentario General 12 menciona que "El derecho a la alimentación adecuada se ejerce cuando todo hombre, mujer o niño, ya sea solo o en común con otros, tiene acceso físico y económico, en todo momento, a una alimentación adecuada o a medios para obtenerla" (Consejo Económico y Social, 1999).

El derecho a la alimentación consiste en tener acceso de manera regular, permanente y libre a una alimentación adecuada y suficiente, que garantice una vida psíquica y física, individual y colectiva, libre de angustias, satisfactoria y digna (Jusidman-Rapoport, 2014). El acceso a los alimentos debe ser físico, económico y adecuado, con el fin de satisfacer las necesidades alimentarias de las personas. Se entiende por necesidades alimentarias; al régimen de alimentación en conjunto aporte una combinación de productos nutritivos para el crecimiento físico y mental para el desarrollo de la persona. La FAO señala que no solo es importante el acceso a los alimentos, sino también, el disfrute de otros derechos fundamentales con los que se vincula, como: la educación, la salud, el acceso a la vivienda digna con servicios básicos como agua potable, drenaje y electricidad, así como contar con esquemas de seguridad social. Los problemas nutricionales que presenta México surgen a causa de diversos factores: precios 
internacionales de los alimentos y la impregnación de micronutrientes por el organismo de las personas.

Conforme a lo expresado en este apartado, la alimentación se entiende como el derecho a alimentarse dignamente, para ello, es necesario que a las personas se le garantice el derecho al mínimo vital de subsistencia, es decir, que pueda allegarse de los elementos necesarios para tener una calidad de vida digna y decorosa. Se entiende como el derecho que protege a toda persona, en contra de toda forma que comprometa su subsistencia física y de vida.

El derecho humano a la alimentación requiere que los Estados proporcionen un entorno propicio en el que las personas puedan desarrollar plenamente su potencial para producir o procurarse una alimentación adecuada para sí mismas y para sus familias. Para comprar alimentos, una persona necesita una base de ingresos adecuada: políticas salariales,

públicas y redes de seguridad social que permitan a los ciudadanos poder realizar su derecho a una alimentación.

Al mismo tiempo, la alimentación se vincula con la sustentabilidad, es decir, con el cuidado del medio ambiente, los recursos naturales son el primordial medio para la producción de alimentos y la base de ingresos de muchas familias. El sistema de producción de la comida y las dietas sustentables forman un papel importante en la garantía de este derecho. Además, pretende la equidad social y un cambio en la relación que tienen los seres humanos con la naturaleza.

Lo anterior, permite expresar que, el derecho a la alimentación es universal, esto es, pertenece a todo el mundo, sin distinción alguna. Conforme al marco legal y a la doctrina, protege a todos los individuos, a grupos vulnerables y discriminados, entre los que se ubican: niños, niñas y adolescentes, personas mayores de edad, personas con discapacidad, mujeres, migrantes y pueblos indígenas.

\subsection{Características de la alimentación sustentable}

La alimentación sustentable es aquella que cumple con ciertas características, que permiten brindarles a las personas o a una población, estabilidad alimentaria. Para poder abordar las características de este tipo de alimentación, es necesario conocer qué es la sustentabilidad, ésta consiste en la capacidad de un sistema para mantener su producción en un nivel aproximadamente igual o mayor que su promedio histórico. Esto implica que 
el sistema debe de satisfacer las necesidades del presente sin afectar las oportunidades de bienestar y calidad de vida de las futuras generaciones.

Cuando se habla de sustentabilidad, se hace referencia a algo que puede sostenerse por sí mismo, específicamente consiste en el equilibrio que existe entre la relación del hombre con los recursos del medio al que pertenece (Bioguía, 2018). Hoy en día los países, comunidades y organizaciones, han fallado en la práctica de medidas sustentables para hacer frente a los problemas de alimentación y seguridad alimentaria. Se tiene un modelo de consumo que ha venido deteriorando la biodiversidad y el medio ambiente.

La Comisión Mundial para el Medio Ambiente y el Desarrollo (CMMAD), establecida por la Organización de las Naciones Unidas (ONU) el 19 de diciembre de 1983, definió el concepto de sustentabilidad como un modo de vida individual que parte de una forma particular hasta llegar de una forma general al desarrollo sustentable como el "desarrollo que satisface las necesidades del presente sin comprometer las capacidades que tienen las futuras generaciones para satisfacer sus propias necesidades" (Organizations of American States, 2012).

Por otra parte, la sustentabilidad comprende ciertas características que la distinguen y la hacen relevante. En primer término, respeta al medio ambiente, el desarrollo de producción y consumo de alimentos no afectan al medio ambiente. Se trata de elegir preferiblemente alimentos orgánicos, de estación o producidos cerca del lugar de compra, evitando así que el transporte requiera tanta energía. El envase que transporta el alimento deberá ser reciclado o reutilizable, permitiendo así que se genere una menor cantidad de desechos.

Otra de las características que la distingue es que debe ser saludable, esto quiere decir, el cuidado en la calidad de los alimentos que se consumen por lo que resulta importante revisar el etiquetado. Mientras menos procesados sean los alimentos, más saludables van a ser para el consumo de las personas. Se busca prevenir enfermedades y disminuir el nivel de obesidad y desnutrición en la población.

\section{SUSTENTABILIDAD PARA ALCANZAR LA SEGURIDAD ALIMENTARIA}

En México la seguridad alimentaria se convierte en un problema de desarrollo sustentable cuando se carece de los medios y recursos naturales para satisfacer las necesidades alimentarias de las generaciones presentes y se comprometen las de futuras generaciones, así como el medio ambiente. 
La alimentación y seguridad alimentaria forman parte de los objetivos del Desarrollo Sostenible (ODS) y de las ciento sesenta y nueve metas, con lo que se busca poner fin a la pobreza, luchar contra la desigualdad y la injusticia, y hacer frente al cambio climático (ONU, s.f). Generan oportunidades para mejorar la calidad de vida de la población en un marco de desarrollo sostenible. Las metas del objetivo dos (Hambre cero) pretende combatir el hambre, lograr la seguridad alimentaria, la mejora de la nutrición y promover la agricultura sostenible.

Así pues, la pobreza es la principal causa de hambre en el mundo, representa uno de los problemas más agudos que enfrentan los países, en particular México, conforme a trascurrido el tiempo, se han implementado diversas políticas con enfoque alimentario para su combate en las últimas décadas; sin embargo, ante el escenario en el que se enfrenta el país, derivado de la contingencia sanitaria por la enfermedad de COVID-19, existe el riesgo de revertir lo que se ha avanzado en las carencias sociales, más en los grupos vulnerables y presentar un incremento en la inseguridad alimentaria.

Entre 2018 y 2020, el porcentaje de la población en situación de pobreza a nivel nacional presentó un incremento de $41.9 \%$ a $43.9 \%$, mientras que el número de personas en esta situación pasó de 51.9 a 55.7 millones de personas. Igualmente, la población en pobreza extrema incrementó de $7.0 \%$ a $8.5 \%$ en dicho periodo y aumentó el número de personas en situación de pobreza extrema del 8.7 a 10.8 millones de personas (Consejo Nacional de Evaluación de la Política de Desarrollo Social, 2021). La pobreza es un fenómeno que se relaciona con las condiciones de vida que vulneran la dignidad de las personas, limitan sus derechos como el de la alimentación e impide la satisfacción de necesidades básicas. La FAO proporciona datos importantes al señalar que casi 767 millones de personas continúan viviendo en la extrema pobreza y aún están generalizadas desigualdades de toda índole (FAO, 2018). La mayoría de la población pobre reside en zonas rurales y depende de la agricultura para su subsistencia y seguridad alimentaria; No obstante, a menudo no pueden beneficiarse de las nuevas tecnologías ni del acceso a los mercados que podría aumentar su productividad e ingresos dado que carecen de recursos o conocimientos para invertir en sus tierras o medios de vida.

En este tenor, el concepto de Seguridad Alimentaria, surge en la década de los setenta, basado en la producción y disponibilidad alimentaria a nivel global y nacional. En los años ochenta se añadió la idea de acceso, tanto económico como físico. Fue hasta la 
década de los noventa que se llegó al concepto actual que incorpora la inocuidad y las preferencias culturales, y se reafirma la Seguridad Alimentaria como un Derecho Humano (PESA, 2011).

Al respecto, la Organización de las Naciones Unidas para la Agricultura y la Alimentación (FAO) menciona que, desde la Cumbre Mundial de la Alimentación de 1996 la Seguridad Alimentaria se da "cuando todas las personas tienen acceso físico, social y económico permanente a alimentos seguros, nutritivos y en cantidad suficiente para satisfacer sus requerimientos nutricionales y preferencias alimentarias, y así poder llevar una vida activa y saludable" (FAO, s.f.). Se convocó como respuesta a la persistencia de una desnutrición generalizada y a la creciente preocupación por la capacidad de la agricultura para cubrir necesidades futuras de alimentos.

La seguridad alimentaria debe ser atendida como la formula del movimiento social de La Vía Campesina, se considera "un proceso político, que implica la toma de conciencia, la acción comunitaria, la reflexión y la acción de los mecanismos de producción e intercambio de alimentos" (Cano Contreras, 2015, pp.70-91). Se debe proteger la capacidad de producir nuestros alimentos y tener mecanismos de intercambio, producción y abastecimiento que no dependan de agentes externos para consumirlos. Se considera una condición necesaria para el disfrute del derecho a la alimentación.

Desde la perspectiva doctrinal, Julieta Sánchez Cano menciona que "el enfoque del derecho a la alimentación complementa los elementos de la seguridad alimentaria relativos a las disponibilidad, acceso, estabilidad y utilización teniendo en cuenta la dignidad humana y la aceptabilidad cultural” (Sánchez Cano, 2014). Esta situación requiere responsabilidad de los gobiernos para cumplir con los compromisos adquiridos en cuanto a seguridad alimentaria, tal y como lo señala Sánchez Cano, de esta forma los ciudadanos dejan de ser objeto de asistencia para convertirse en sujeto de derecho.

La seguridad alimentaria se comprende como una condición necesaria para el disfrute del derecho de alimentación, definiéndose como "un estado donde está garantizado el acceso de todas las personas, en todo momento a los alimentos necesarios, seguros y nutritivos para llevar una vida sana" (Menéndez Rexach, 2015, p. 50). Es entendida como seguridad del abastecimiento alimentario.

Para Edelman el concepto de seguridad alimentaria tiene origen en los años ochenta en un "programa de alimentación del gobierno mexicano, que no buscaba únicamente la 
autosuficiencia alimentaria, también el control nacional de las cadenas de producción, con el fin de reducir la dependencia de los productos extranjeros” (Edelman, Marc, 2018). El autor no hace referencia a un concepto de soberanía alimentaria, pero señala que las regulaciones a la producción que proponen algunos defensores de la misma pueden limitar la acción de los productores campesinos al restringir las exportaciones e importaciones y controlar los tipos de explotación agrícola, yendo en contra del concepto en mención.

Cuando no se cuenta con los medios monetarios para los alimentos suficientes y necesarios, la seguridad alimentaria pasa a convertirse en un problema de desarrollo económico. La principal meta en la que se enfoca la FAO, es asegurar que todas las personas puedan acceder regularmente a una cantidad suficiente de alimentos de calidad que les permita a las personas llevar una vida activa y saludable.

La seguridad alimentaria es considerada como un modelo alimentario que enfatiza los problemas de inseguridad que existen en los hogares, en las personas y en los países que dependen de las importaciones para cubrir las necesidades básicas de sus habitantes; el no contar con suficiente abastecimiento de alimentos, ni el acceso a los recursos adecuados como el empleo y la estabilidad de tener una alimentación, inciden a vivir en carencia, en un estado de hambre y pobreza tiene como consecuencia el no poder garantizar el derecho humano a la alimentación de las personas.

La mayor concentración de pobreza e inseguridad alimentaria se sigue observando en zonas rurales, donde la población depende directa o indirectamente de la agricultura, para obtener tanto ingresos como alimentos; sin embargo, son los que hoy en día se encuentran en desigualdad y lejos de tener acceso a una alimentación diga y saludable, con la que cubran sus necesidades alimentarias.

En tal sentido, la gran parte de la población rural pobre está formada por agricultores familiares, productores de subsistencia o trabajadores agrícolas sin tierras, incluyendo a personas que dependen del bosque con acceso limitado a los medios de producción.(FAO, 2020). Son más dependientes las familias rurales de los ingresos de tipo no agrícola, debido a que no cuentan con los recursos, ni con las infraestructuras y los servicios para obtener los créditos e invertir en el campo. La población pobre es la más vulnerable.

Además, los agricultores familiares dependen de la agricultura como actividad primaria y de los recursos naturales para su subsistencia, se integran en redes territoriales y 
culturales con el fin de generar empleo en zonas agrícolas y rurales, su participación en fundamental para el crecimiento de la economía del país, combatir el hambre y la pobreza, así como garantizar la alimentación de las poblaciones.

La vulnerabilidad implica la presencia de factores que inciden en la seguridad alimentaria, es decir, factores que exponen a la población el riesgo de sufrir inseguridad alimentaria, al no contar con suficientes alimentos o no poder acceder de manera permanente a los mismos, e incluso, el no tener la información sobre aspectos nutricionales o que carecen de un trato preferente en el ámbito del derecho a la alimentación se convierte en un problema vinculado con el desarrollo sustentable.

Los alimentos y la agricultura también son una de las grandes prioridades de la Agenda 2030. La mayoría de los 17 ODS contienen objetivos vinculados directa o indirectamente con la seguridad alimentaria y la nutrición. Las medidas para erradicar el hambre y la pobreza, alcanzar la seguridad alimentaria y mejorar la nutrición constituyen inversiones cruciales en la salud y el bienestar del ser humano; inversiones que son vitales para llevar a cabo la Agenda 2030 de forma plena.

En México, la agricultura es considerada como una de las actividades con mayor relevancia debido a que genera gran cantidad de empleos en el país, es el sector productivo más importante desde el punto de vista económico, social y ambiental, de ésta depende la alimentación primaria de las personas, el incremento de la población productiva y la preservación y cuidado del entorno. Es una base significativa para el desarrollo del país ya que ayuda a fomentar la seguridad alimentaria.

Dado lo anterior, resulta más benefactor invertir en la agricultura, su repercusión en la reducción de la pobreza resulta mayor que en la inversión en otros sectores, ya que ofrece los medios para que la población rural se beneficie de sus principales activos: tierra y mano de obra. Sería una forma de garantizarle no solo la disponibilidad, sino el acceso a los alimentos básicos para su sobrevivencia. No se omite resaltar que las personas en pobreza y en estado de vulnerabilidad son a las que menos les llega los recursos y los medios necesarios para poder tener una alimentación más adecuada a sus necesidades, siendo objetos de una desigualdad total.

La reducción de la pobreza rural también requiere aumentar la productividad de las pequeñas explotaciones, promover cadenas de valor más eficientes, crear empleo, especialmente para los jóvenes, fomentar la diversificación económica, invertir en las 
personas y fomentar competencias que puedan usarse en actividades agrícolas y no agrícolas., programas de desarrollo social con un enfoque de derechos humanos y universales, que sean efectivos para que las personas en pobreza y vulnerabilidad puedan gozar de ese derecho como garantía al derecho a la alimentación.

Por otra parte, la Asamblea General de la ONU en 1997, reconoció que proporcionar seguridad alimentaria sustentable constituye el desafío más grande que enfrenta la humanidad. La sustentabilidad alimentaria no sólo se restringe a la productividad, sino a una serie de actividades que giran alrededor de quienes buscan obtener y acceder a los alimentos, como lo es el hambre y la desnutrición ya que algunas personas sufren de estos problemas, mientras que en otros lugares existen personas que desperdician alimento, que en muchos casos estos podrían ayudar a personas que sufren de esta limitación.

La sustentabilidad alimentaria es importante, debido a que muchos recursos energéticos son consumidos en el mundo en función de la alimentación del ser humano; todos los productos que son llevados a nuestra mesa ocuparon energía durante su producción, elaboración y transporte. Esto lleva a considerar que una sociedad que se preocupa por fomentar un desarrollo económico mediante la conservación del medio ambiente, en un ámbito de paz, igualdad y respeto a los derechos humanos, es una sociedad próspera que busca ser mejor mediante un desarrollo sustentable.

El hambre es uno de los problemas latentes en México, se define como la escasez de alimentos básicos, que causa carestía y miseria generalizada (Real Academia Española, 2017). Se relaciona con el concepto de seguridad alimentaria. Según el Programa Mundial de Alimentos alrededor de 135 millones de personas padecen hambre severa, debido a los conflictos causados por los seres humanos, cambio climático y las recesiones económicas (Guterres António, 2020, p.2). Ahora con la pandemia de Covid-19 podría duplicar dicha cifra, poniendo en riesgo a millones de personas que carecen de los recursos y medios para cubrir sus necesidades alimentarias.

Tanto la desnutrición como las condiciones de hambre en las que se encuentra una población, no tan sólo son problemas sociales que comportan una parte considerable de costos económicos, incluyendo una reducción de productividad y un uso insostenible de los recursos. El hambre pone en marcha un círculo vicioso de menor productividad y como consecuencia, la existencia en México de un número significativo de personas en pobreza; un lento desarrollo económico y degradación de los recursos naturales. 
Uno de los aspectos de la sustentabilidad que se observa en los procesos productivos, es que contribuye en mejorar la calidad de vida de los seres humanos, apoya en resolver problemas que se presentan como la pobreza, el cambio climático y a mejorar la relación entre la naturaleza y el hombre, con el fin de tener una adecuada gestión ambiental y social.

\section{CONCLUSIONES}

Para lograr la seguridad alimentaria se requiere el suministro suficiente de alimentos en los hogares de México. Este modelo alimentario se relaciona con la salud de las personas y dentro de ella se ubica la sustentabilidad, quien se vincula con varios aspectos importantes como: los procesos de producción, sus consecuencias ambientales y sociales. Mientras aún existan millones de personas que sufran hambre y vivan en extrema pobreza, no puede haber alimentación sustentable. Los agricultores pobres se preocupan seriamente por los limitados recursos de los cuales dependen. Debido a la falta de acceso suficiente a la tierra, al agua y a tecnologías adecuadas, sus acciones se ven dominadas por la lucha por sobrevivir.

La agricultura sustentable y el desarrollo rural son la base para el éxito en la lucha contra el hambre y la pobreza. La baja producción agrícola y la degradación de los recursos naturales atentan contra la seguridad alimentaria. Si se quiere alcanzar el compromiso de la comunidad internacional, en cuanto a garantizarles a las personas una alimentación adecuada y contar con los elementos mínimos para su sobrevivencia, es esencial evitar el impacto ambiental, así como contar con sistemas alimentarios sustentables que garantizan la seguridad alimentaria y la nutrición de las personas.

\section{REFERENCIAS BIBLIOGRAFICAS}

Constitución Política de los Estados Unidos Mexicanos (CPEUM). Artículo 27 Fracción XX, última reforma publicada en el Diario Oficial de la Federación el 27 de agosto de 2018. (México)

Congreso Constituyente (21 de junio de 2018) Ley General de Salud. Organización de las

Naciones Unidas para la Alimentación y la Agricultura (2019), El trabajo de la FAO sobre el derecho a la alimentación, FAO, http://www.fao.org/3/ca6142es/ca6142es.pdf.

Barriguete Meléndez, J. Armando (2017), Para entender la alimentación, Nostra, (2 2 ed.), México, (p. 25). 
Naciones Unidas, Derechos Humanos, FAO, El derecho a la alimentación adecuada, Folleto informativo no. 34, (p. http://www.fao.org/docrep/016/b358s/b358s.pdf.

Artículo 25 de la Declaración Universal de Derechos Humanos. adoptada y proclamada por la Asamblea General en su resolución 217 A (III), de 10 de diciembre de 1948.

Consejo Económico y Social, El derecho a una alimentación adecuada (art. 11), 12/05/99. Observación general: 12, Naciones Unidas, 12 de mayo de 1999, https://www.acnur.org/fileadmin/Documentos/BDL/2001/1450.pdf.

Jusidman-Rapoport, Clara (2014) “El derecho a la alimentación como derecho humano", Salud Pública de México, (vol. 56, suplemento 1)

Bioguía, Sustentabilidad: definición y concepto, consulta: 02 de octubre de 2018, https://www.bioguia.com/notas/que-significa-la-sustentabilidad.

Organization of American States http://www.oas.org/dsd/publications/Unit/oea06s/ch03.htm (Recuperado el 21 de diciembre de 2012).

ONU México, Ban Ki-moon, Secretario General de las Naciones Unidas (2006-2016), Agenda 2030, http://www.onu.org.mx/agenda-2030/.

Consejo Nacional de Evaluación de la Política de Desarrollo Social, Estimaciones de pobreza multidimensional 2018 y 2020, Comunicado, México, (05 de agosto de 2021).https://www.coneval.org.mx/SalaPrensa/Comunicadosprensa/Documents/ 2021/COMUNICADO_009_MEDICION_POBREZA_2020.pdf.

Organización de las Naciones Unidas para la Alimentación y la Agricultura (FAO, 2018), Pobreza y hambre, diferentes pero conectados, http://www.fao.org/about/whatwe-do/so3/es/.

Programa Especial para la Seguridad Alimentaria (PESA) en Centroamérica (2011), Proyecto Food Facility Honduras, Seguridad Alimentaria Nutricional. Conceptos básicos (3a. ed.) (p. 2), consulta 17 de abril 2014: http://www.fao.org/3/aat772s.pdf.

La Organización de las Naciones Unidas para la Agricultura y la Alimentación (FAO), http://www.fao.org/economic/ess/ess-fs/es/.

Cano Contreras, Eréndira Juanita, (diciembre 2015), "Huertos Familiares: Un camino hacia la seguridad alimentaria”, vol. 10, (núm. 20), Revista Pueblos y Fronteras 
Digital, Universidad Nacional Autónoma de México, Distrito Federal, México, (pp. 70-91).

Sánchez Cano, Julieta Evangelina et. Al (enero-junio 2014), "La importancia de la gobernanza en la seguridad alimentaria ante un panorama de volatilidad en el principio internacional de los alimentos; el caso de México", año1, núm 2. Revista Perfiles de las Ciencias Sociales, Universidad Juárez Autónoma de Tabasco (UJAT), México.

Menéndez Rexach, Ángel, (2015), Estudios jurídicos sobre seguridad alimentaria, Madrid, Marcial Pons, (p. 50)

Edelman, Marc (enero-junio 2018), "Estudios agrarios críticos: tierras, semillas, soberanía alimentaria y derechos de las y los campesinos”, vol. 54, (núm. 1), Revista Colombiana de Antropología, Quito: Instituto de Altos Estudios Nacionales (IAEN).

FAO (2020), Reducirla pobreza rural, http://www.fao.org/reduce-ruralpoverty/overview/quien-forma-la-poblacion-rural-pobreles/.

Diccionario de la Real Academia Española, (2017) Edición del Tricentenario, http://dle.rae.es/?id=K02yR30.

Guterres, António, Global reporto $n$ food crises (2020), Joint analysis for better decisions, Food Security Information Networw, (p.2) https://docs.wfp.org/api/documents/WFP-

0000114546/download/?_ga=2.101197697.188447121.1629671167689694500. 1604859829. 